\title{
La investigación como eje transversal en la carrera de contabilidad. Un estudio de caso en Ecuador*
}

\author{
[Artículos]
}

\author{
Marcelo Mantilla-Falcón** \\ Jessica Gavilanes-Sánchez ${ }^{* * *}$ \\ Karina Benítez-Gaibor****
}

Recibido: 27 de abril de 2021

Aprobado: 15 de junio de 2021

Citar como:

Mantilla, M., Gavilanes, J. y Benítez, K. (2021). La investigación como eje transversal en la carrera de contabilidad. Un estudio de caso en Ecuador. Revista Activos, 19(1).

https://doi.org/10.15332/25005278.6689

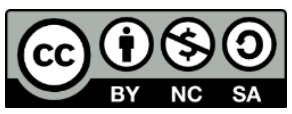

\section{Resumen}

El presente estudio identifica el nivel de apropiación de la investigación en la formación académica de los estudiantes de los últimos semestres de la carrera de Contabilidad y Auditoría en una institución de educación

\footnotetext{
* Investigación derivada de trabajo de titulación profesional en Contabilidad y Auditoría ** Doctor en Ciencias de la Educación. Docente-investigador de la Universidad Técnica de Ambato, Ecuador. Correo electrónico: luismmantilla@uta.edu.ec; ORCID: https://orcid.org/0000-00028209-7365

${ }^{* * *}$ Ingeniera en Contabilidad y Auditoría. Universidad Técnica de Ambato, Ecuador. Correo electrónico: jgavilanes0793@uta.edu.ec; ORCID: https://orcid.org/0000-0002-7926-1718 ${ }^{* * * *}$ Doctora en Contabilidad y Auditoría. Docente-investigadora de la Universidad Técnica de Ambato, Ecuador. Correo electrónico: marcelakbenitez@uta.edu.ec; ORCID: https://orcid.org/0000-0002-5214-9208
}

\section{Revista Activos}

ISSN: 0124-5805 | e-ISSN: 2500-5278 | DOI: https://doi.org/10.15332/25005278

Vol. 19 N.o 1 | enero-junio de 2021 
superior en Ecuador. Se trata de una investigación cuantitativa, descriptiva, transversal, con muestreo no probabilístico de carácter intencional. Participaron 118 estudiantes de octavo y noveno semestre. Para la recolección de información, se aplicó una encuesta estructurada con preguntas dicotómicas, opción múltiple y escala de Likert; en el análisis estadístico se utilizó el coeficiente de correlación de Pearson y Ji cuadrado; además, se usaron descripciones a nivel porcentual. Los resultados evidencian que la investigación no es una fortaleza en la formación académica de los estudiantes de la carrera; sin embargo, utilizan sus herramientas a la hora de realizar sus trabajos de titulación.

Palabras clave: investigación, contabilidad, formación profesional, perfil de egreso.

Clasificación JEL: M49.

\section{Research as a transversal axis in the accounting career. A case study in Ecuador}

\section{Abstract}

This study identifies the level of appropriation of research in the academic training of students in the last semesters of the Accounting and Auditing Career in a higher education institution in Ecuador. It is a quantitative, descriptive, cross-sectional research with non-probabilistic intentional sampling. A total of 118 eighth and ninth semester students participated. To collect information, a structured survey was applied with dichotomous questions, multiple choice and Likert scale; the statistical analysis used Pearson's correlation coefficient and Chi square; in addition, descriptions at the percentage level were used. The results show that research is not a strength in the academic training of students of the career; however, they use its tools when making their graduation project.

Keywords: research, accounting, professional training, graduate profile.

JEL classification: M49. 


\section{Introducción}

En este apartado se pretende describir cómo la actividad científica está inmersa en la formación del contador público, de modo que se encuentre un conocimiento completo que se exteriorice a través de habilidades, competencias o destrezas: "El conocimiento puede ser definido como una reproducción conceptual de la realidad, y, como tal, como una elaboración que se produce en el cerebro de los seres humanos, una formulación intelectual” (Ortiz-Ocaña, 2016, p. 8).

En la misma línea de pensamiento de Ortiz-Ocaña, se pretende buscar nuevos escenarios donde la contabilidad se desarrolle desde una perspectiva diferente y producir conocimiento científico. No obstante, esta actividad cada vez se vuelve más compleja, pues su finalidad ha sido controlar el recurso económico a través de procedimientos técnicos, teóricos y prácticos; por tanto, ha surgido la necesidad de involucrar una reflexión científica en la contabilidad, donde jueguen un papel importante el conocimiento, el rol del estudiante y el rol de profesional contable.

El objetivo de este estudio es determinar la influencia de la investigación en la formación de los estudiantes que se encuentran cursando los últimos niveles de la carrera de Contabilidad y Auditoría de la Universidad Técnica de Ambato (UTA), Ecuador. Con esto, se busca analizar el perfil público del contador ecuatoriano que sirva como referente para la misma institución, para las nuevas generaciones y para los profesionales en ejercicio vigente.

La experiencia de los investigadores y la labor docente ejecutada a través de los años, permite percibir que la actividad investigativa en las aulas universitarias de los futuros contadores no es tratada con profundidad y no se convierten en un eje trasversal a lo largo de la carrera, solo se vuelve a tocar el tema de investigación cuando el estudiante se avoca al desarrollo 
de su trabajo de graduación. Con estos referentes, surge la interrogante sobre la relación, repercusión o influencia que tiene la investigación en el perfil de egreso y formación profesional del futuro contador ecuatoriano.

\section{Antecedentes}

El presente estudio busca describir y comprender las fortalezas investigativas de los estudiantes de la carrera de Contabilidad y la aplicación que le dan a la metodología de la investigación científica en la formación educativa.

La notable evolución conceptual de la contabilidad en los últimos años y el estado actual de su discusión académica representa un conflicto para llegar a un acuerdo en términos epistemológicos y ontológicos, puesto que en las carreras contables se dedica más tiempo a tecnificar y mecanizar los procesos de registro de cuentas y menos al análisis y abstracción de su utilidad científica. A partir de esto, Ordoñez-Noreña (2008) señala que la formación del estudiante contador se orienta más a la elaboración de tareas prácticas, que no requieren suficiente esfuerzo intelectual y de desarrollo de conocimiento, lo que la hace una educación orientada más al hacer que al saber. Por ende, es importante considerar los graves problemas que a nivel de Latinoamérica existe en cuanto al desarrollo de la ciencia y tecnología (Quintanilla, 2008).

Visto así, la producción investigativa en contabilidad, y en cualquier disciplina, requiere de elementos interdisciplinares y, sobre todo, metodológicos para que esta se pueda llevar a cabo. Esto demanda un direccionamiento de cursos de metodología hacia la capacitación en herramientas de investigación, con la finalidad de ampliar los horizontes de la contabilidad y de construir, desde los avances profesionales, un mecanismo de cambio organizacional y de aporte social a través de una investigación seria y rigurosa (Gómez, 2003). 
Abordando lo anterior, Quinche (2006) menciona que, en la disciplina contable, existen muy pocas personas que se dedican a la investigación histórica contable, que desde hace tiempo "ha sido concebida como la recolección de datos, fechas, cantidades, valores, etc., relacionados en un espacio y tiempo; es decir, que se limita a la visión de hechos, la mayor parte aislados, ocurridos alrededor del hombre y su acción” (p. 189). En este sentido, es importante conocer que la contabilidad tiene su propia historia y desarrollo a nivel mundial, lo cual servirá para consolidarse dentro del campo científico y por ende, en la formación y desempeño profesional.

Según Bahía (2004) en la investigación contable se usan algunos métodos que han ayudado a generar conocimientos, a través de la práctica y del empirismo. Sin embargo, ante la imposibilidad de guardar las transacciones en la memoria, apareció la necesidad de llevar registros y así se desarrolló la evolución del pensamiento contable, con lo cual se crearon programas de investigación que ayudaran al avance de la ciencia y de la disciplina.

Por su parte, Casal y Viloria (2007) relacionan al pragmatismo y materialismo como una influencia muy significativa en el pensamiento contable, pues manifiestan que lo que es verdadero es construido por lo que realmente es útil, práctico, concreto y productivo. De esta manera, los avances de la ciencia y la tecnología de la información son una ayuda para la contabilidad, al proyectar y consolidar el ejercicio profesional avanzando hacia la construcción de la ciencia contable a través de la investigación.

Según el estudio de Pinzón (2011), hoy en día las universidades continúan viviendo sus crisis porque cada vez hay más competencias y más exigencias por parte del Estado. Justamente con las pruebas, la 
acreditación de calidad y demás requerimientos externos, se ha logrado crear grupos de investigación, pero no se relacionan lo suficiente con procesos reales de producción científica y producción académica.

En lo que respecta a la formación para la investigación, Ríos (2012) menciona que se alcanza a través de actividades concretas en programas específicos de investigación formativa que faciliten el paso hacia la aplicada. A esta última la antecede un proceso de apropiación de la cultura investigativa, que incorpora un entrenamiento para los estudiantes y desarrolla estrategias tendientes a aprender a formular preguntas, generar hábitos de lectura e identificar los modos para acceder a fuentes de búsqueda de información.

Con esto se busca que los programas de formación indaguen cómo el contador público adquiere competencias cognitivas, socioafectivas y comunicativas necesarias para localizar, extraer y analizar datos provenientes de múltiples fuentes, que ayudarán al estudiante a obtener una adecuada formación para la investigación. Finalmente, los procesos de aprendizaje en temas de la disciplina contable cobran importancia a medida que van integrando los saberes que va adquiriendo el estudiante en el desarrollo de su carrera, siempre y cuando tengan el acompañamiento de docentes comprometidos con la actividad científica que aporten al área de conocimiento.

Desde el punto de vista de Macías (2013) hay varios estudios planteados donde el trabajo de campo en investigación contable no es un tema nuevo. Existen contribuciones que hacen mención a la incorporación de aspectos empíricos en la contabilidad de gestión, así como trabajos de campo en investigación contable, donde se señala que estas investigaciones han permitido identificar prácticas de vanguardia y mejorar la comprensión del fenómeno, así como vincular la práctica con la investigación. 
Po otro lado, es necesario preguntarse constantemente si existen nuevas alternativas para la educación y formación de los profesionales en Contaduría Pública, con la finalidad de contribuir a su formación con un alto grado de excelencia académica y profesional, a través de las prácticas sobre las cuales se forman los estudiantes. Es importante revisar los preceptos sobre los que se enseña no solo la contabilidad sino cada disciplina en general. Para esto, se requiere utilizar métodos, técnicas e instrumentos de investigación, con el fin de buscar un acercamiento comprensivo y crítico del área contable al escenario académico y a los problemas de la sociedad; así, los profesionales contables pueden ser sujetos capaces de pensar, comprender y transformar la realidad, por lo que la posibilidad de hacerlo brindaría una educación reflexiva y crítica (Gómez, 2013).

A partir de aquello, es importante considerar que "en América Latina la investigación contable representa un problema grave" (Saavedra y Saavedra, 2015, p. 100). De esta manera, los países que la conforman tienen limitada inserción en la economía mundial, pues no existe suficiente personal que se dedique al ámbito y tampoco ha habido inversión, ni tecnología necesaria para los programas de contabilidad que han iniciado sus procesos en el tema. Sin embargo, "han existido intentos muy poco significativos que impliquen el desarrollo de una didáctica especifica de la disciplina contable" (García, 2017, p. 123), ya sea como contenidos teóricos como en el campo del saber; por esto, en términos de lo contable, es posible declararlo un campo inexplorado. Por su parte, Gallegos y Rodríguez (2017) mencionan que "la actividad científica y por ende la investigación y su relación con el ámbito contable resulta muy poco productivo" (p. 17). Por ello, Villareal y Córdoba (2017) mencionan que es importante reflexionar sobre la evolución del pensamiento contable como parte de la formación profesional, con el fin de que los nuevos estudiantes 
se vayan formando a través de una investigación crítica e interpretativa que permita buscar nuevos conocimientos.

En vista de lo anterior, es significativo tomar un aporte de Mantilla-Falcón et al.(2018), quienes mencionan que es "responsabilidad y compromiso de las instituciones de educación superior formar profesionales con competencias y destrezas adecuadas para el desempeño profesional, así como el poder desenvolverse con eficiencia, eficacia e idoneidad" (p. 92). Sin embargo, la formación contable, ya sea a nivel nacional como a nivel internacional, debe ser completa en todos los sentidos, sea en conocimientos prácticos y teóricos, así como el desarrollar principios y valores tanto profesionales y personales.

En ese sentido, en estudio de Cervantes et al. (2018) buscaron aportar a la comunidad una reflexión en torno a la ciencia contable para que pueda ser vista desde su propia historia y evolución, con el fin de brindar a quienes opten por formarse en este ámbito, capacidad de responder a problemas frecuentes dentro de las organizaciones y de la sociedad.

Con el propósito de analizar el perfil del contador público desde su ámbito profesional, Fernández et al.(2019) realizaron un estudio en Colombia, Ecuador, México, Perú y Venezuela donde concluyeron que el diseño curricular en las instituciones de educación superior guarda grandes diferencias, debido a que es una carrera que conlleva trabajo independiente y responsable. Sin embargo, algunas universidades son más innovadoras que otras, pues consideran que el diseño curricular está soportado por bases muy consistentes para construir una propuesta innovadora en materia contable, además de generadora de conocimiento que esté asociado a la experiencia, objetividad, independencia y actuar ético, lo cual incide de forma exitosa en el desempeño laboral de estos profesionales. 


\section{Metodología}

Se trata de una investigación de corte cuantitativo y descriptiva, puesto que "busca especificar propiedades y características importantes de cualquier fenómeno que se analice. Describe tendencias de un grupo o población” (Hernandez et al., 2014, p. 92). En efecto, se detallan las percepciones que tienen los estudiantes con respecto a la investigación en su formación académica. Es un estudio transversal puesto que se aplicó el instrumento por una sola vez; su muestreo es no probabilístico de carácter intencional, a criterio de los autores.

Participaron en este estudio 118 estudiantes de los últimos semestres de la carrera de Contabilidad y Auditoría de la Universidad Técnica de Ambato, UTA, $44.06 \%$ de octavo y 55.94 \% de noveno semestres. El criterio para trabajar con estos niveles obedece a que muchos de los estudiantes ya se encuentran en el campo laboral (50.8 \%) y tienen un criterio más formado de la práctica profesional fuera de las aulas. Como es una constante en esta carrera, el género femenino tiene una mayor participación en el total con el $72.89 \%$, y el masculino representado a penas con el $27.11 \%$ (MantillaFalcón et al., 2017).

La UTA tiene más de 52 años de existencia y es el mismo tiempo que tiene la carrera de Contabilidad y Auditoría, lo que la hace una de las más antiguas en el Ecuador. En el transcurso de la formación del profesional contador, reciben la asignatura de Metodología de la Investigación en primer semestre con una duración de 3 créditos equivalentes a 48 horas presenciales y 96 horas de trabajo autónomo y experiencial sumando 144 horas de preparación. En el penúltimo semestre (noveno) reciben Diseño de Proyectos de Investigación con un total de 48 horas presenciales y 72 horas de trabajo autónomo, lo que suma 120 horas en total dedicadas a la estructuración de su trabajo final de graduación (tesis). 
La información se recogió mediante una encuesta estructurada con preguntas de tipo dicotómica, opción múltiple y escala de Likert, con opciones de respuesta: (5) totalmente de acuerdo; (4) de acuerdo; (3) ni de acuerdo ni en desacuerdo; (2) en desacuerdo, y (1) totalmente en desacuerdo. Esta última se dividió en tres grupos: el primero recoge la opinión sobre la influencia de las asignaturas recibidas durante la carrera y el impacto en la profesión (Alfa de Cronbach de 0,955); el segundo bloque, sobre el desarrollo profesional y oportunidad de formación $(\alpha=0,969) ; y$, finalmente el tercer bloque sobre las capacidades profesionales $(\alpha=$ 0,962). Los estudiantes presentaron edades entre los 21 y 32 años con una $\overline{\mathrm{X}}=23,78$ años y una DS de 2,12. Estadísticamente se utilizó " $r$ " de Pearson, para medir correlaciones, Ji cuadrado para asociaciones de variables y análisis porcentual de los datos.

\section{Resultados}

Este estudio trata de abordar los conocimientos, destrezas y habilidades que los estudiantes, al culminar su carrera, poseen sobre investigación, los resultados no son muy alentadores: Bajo (5,1\%); Regular (35,6 \%) y Medio (59,3 \%); esta información es el claro reflejo que la investigación no es una fortaleza en la carrera de contabilidad puesto que su diseño y malla curricular es mayormente profesionalizante pues el egresado busca inmediatamente enrolarse al mundo laboral como auxiliar, asistente o personal de apoyo en el área contable, tributaria o de costos. Por tanto, necesita poner mayor atención a las asignaturas que le forman para cumplir este rol en el mundo laboral. La investigación solo es un medio para estructurar su trabajo de titulación.

De acuerdo con lo dicho por Colina (2007), es importante que los estudiantes universitarios, a través de programas curriculares, desarrollen 
capacidades científicas para aprender a aprender, construir y resolver los problemas atenientes a la profesión y la realidad social circundante.

Desde el punto de vista semántico, no tienen claro el concepto mismo de la investigación, que la entiende como: "Un conjunto de procesos sistemáticos, críticos y empíricos que se aplican al estudio de un fenómeno o problema" (Hernandez et al., 2014, p. 4). El 1,7 \% considera que es un proceso intelectual; $15,3 \%$ define como un conjunto de métodos sistemáticos; $38,1 \%$ cree que es un mecanismo para obtener nuevos conocimientos y finalmente; $44,9 \%$ asegura que es una actividad para generar conocimiento científico. Estos indicadores dejan ver que hay confusión y poca claridad en lo que metodológicamente representa la investigación científica; por tanto, "es clave que ellos entiendan la necesidad de formarse para iniciar procesos de investigación y transformar verdaderas problemáticas que se presentan en la vida cotidiana" (Cifuentes y Pedraza, 2017, p. 49).

$\mathrm{Al}$ inquirir sobre el concepto de 'investigación contable' de manera puntual, la confusión es mayor, aunque el 62,7 \% asegura tener clara la definición; sin embargo, las respuestas se distribuyen así: estudio de la teoría contable, $22 \%$; indagación del comportamiento de eventos financieros, 46,6\%; producción de nuevos conocimientos, 8,o \%; y, instrumentos que rigen el estudio de contabilidad, 24,6\%. Simplemente es importante reconocer que la investigación contable cumple con todos los requisitos comunes a toda investigación científica, pero básicamente "es uno de los medios existentes para lograr la dinamización de los procesos de cualificación, desarrollo y crecimiento tanto social como económico" (Ortiz-Ocaña, 2016, p. 18).

Lo anterior demuestra que los estudiantes tienen la idea de Pérez y Pinto Perry (2016) según la cual la investigación contable se refiere al estudio de 
la teoría contable y a la indagación del comportamiento de eventos financieros, es decir, es la búsqueda metódica, racional y objetiva de conocimientos que permitan la descripción, explicación y control de fenómenos que sean de naturaleza financiera. Con esto se busca que el estudiante realice aportes para el desarrollo de sus habilidades y con fines de aprendizaje constructivo con lo cual podrá aprender a aprender.

La academia aún no se pone de acuerdo en considerar si la contabilidad es una ciencia, un arte, una técnica por eso los estudiantes tienen diferentes apreciaciones y matices que son como sigue: técnica, $43.2 \%$; ciencia, $34.7 \%$; sistema, el $17.8 \%$; arte, el $4.2 \%$. A partir de esto, se puede evidenciar que aún no se ha llegado a una definición exacta y acertada sobre la contabilidad, pues la mayor parte le siguen considerando como una técnica porque deduce cómo se realizan los procesos dentro de una entidad. Sin embargo, la investigación está presente en la contabilidad, pero lo que realmente se busca es que los alumnos la consideren como una ciencia puesto que en la práctica contable se utilizan métodos reflexivos controlados y críticos que garantizan la solución de un problema de manera objetiva, válida y confiable (Castro, 2016).

$\mathrm{Al}$ hablar de los fines o intereses de la contabilidad, el $41.5 \%$ considera que se ocupa de estudiar los hechos económicos de una organización; el 27.1\% se decanta por el registro de las operaciones económicas que realiza una entidad; el 25.4 \% dice que la contabilidad estudia la realidad económica y financiera de las organizaciones, y, finalmente, un $5.9 \%$ manifiesta que se orienta a administrar los gastos e ingresos de una compañía. La mayor parte coincide con lo que argumenta Gonzales (2003): "La contabilidad es una ciencia que mide y registra los hechos económicos ocurridos en la unidad económica denominada empresa”. 
Centrándose el estudio en algo muy concreto sobre las normas y principios de la contabilidad, la percepción de los estudiantes es bastante dispar: nivel bajo, 1,7 \%; regular, 11,9 \%; medio, 62,7 \%; alto, 19,5\%; y, muy alto 4,2 \% (en escala de 1 a 5 ), esto determina que, pese a recibir estos contenidos en la formación académica, no hay una apropiación firme de la teoría específica que, a futuro, se convertirá en el soporte de su profesión y su desempeño profesional. Es importante decir que los principios

[...] son aquellas prácticas o postulados que se derivan de la práctica y de la razón que hacen posible una rendición de cuentas adecuada e imparcial de fácil interpretación, porque constituyen prácticas adoptadas y aplicadas sistemática y consistentemente en casos similares por personas capacitadas en la materia. (Merino et al., 2019, p. 29).

La duda aquí es si la educación y la enseñanza de la contabilidad está fallando. De ser así, es importante tomar en cuenta la recomendación de Cabeza y Castillo (2005):

La enseñanza de la contabilidad debe partir de la presentación de situaciones reales, pasar a la conceptualización y continuar con talleres, prácticas, casos que concreten los conceptos en escenarios simulados de realidades, y después ir a la realidad misma para confrontar las conceptualizaciones con la vida real. (p. 86)

Contrastando un fenómeno epistemológico entre contabilidad y conocimiento, las respuestas son bastante equitativas al expresar que la contabilidad busca una respuesta a la necesidad de poseer información (37,3 \%); que el conocimiento contable mejora la capacidad de generar ventajas competitivas sostenibles (33,9\%), y aporta verdadero valor a las organizaciones (28,8 \%). En definitiva, como dice Baena (2017), el conocimiento se adquiere de diversas maneras al ser considerada como una habilidad humana que dará respuestas a las cuestiones que el ser 
humano pueda hacerse, en este caso en el campo de la contabilidad y la empresa.

Es generalizada la percepción de que la contabilidad debe ser una asignatura que se debe aprender de manera teórico-práctica como así lo aseguran el 68,6 \%, y mayormente práctica, 27,1\%. En definitiva, toda aplicación debe partir de una teoría y toda teoría se refuerza con la práctica. Esto significa otro reto para la clase docente de fundamentarse en didácticas orientadas a una pedagogía práctica de la contabilidad para garantizar su asimilación y comprensión holística (Cabeza y Castillón, 2005).

La visión generalizada de la aplicabilidad de la investigación en la actividad académica está direccionada a la construcción de proyectos (48.3 \%) y otro porcentaje parecido en el desarrollo de tareas (40.7 \%). Sin embargo, al concluir la carrera, la mayoría de estudiantes debe elaborar un trabajo de fin de grado denominado "proyecto de investigación", donde convergen los recursos metodológicos de la investigación científica por obligación y requisito. Aquí, se evidencia la débil cultura investigativa durante la formación académica y profesional; de hecho, queda más evidente en el campo laboral, donde escasamente ponen en juego habilidades investigativas por ser una carrera estrictamente profesionalizante.

Del poco trabajo investigativo llevado a cabo en la carrera, las modalidades más usadas en la investigación es la bibliográfica-documental (fuentes secundarias) con más del $66 \%$; muy poco se realiza investigación de campo (26.3\%), es decir, desde la fuente primaria, en este caso, la empresa, sabiendo que esta es la generadora de datos financieros para la toma de decisiones en la que se basan los gerentes y administradores 
(González y Bermúdez, 2010). El trabajo de campo y la investigación in situ sigue siendo una prioridad dentro de la academia.

$\mathrm{Al}$ cuestionar sobre el nivel de profundidad o tipo de investigación realizadas, las más aplicadas son la exploratoria, con el 16,9\%, y la descriptiva, con el 20,3\%; más del $50 \%$ no responde a esta pregunta, precisamente por el desconocimiento de los conceptos y alcances de los estudios existentes en el campo de la metodología de la investigación. Al parecer, los estudios contables giran en torno a estos dos tipos de investigación y también al estudio de casos bajo la modalidad exploratoria y descriptiva únicamente (Segarra et al., 2016).

Bajo esta perspectiva los estudiantes tienen muy claro qué asignaturas fueron muy influyentes en la formación profesional y están aportando para el campo laboral, en los casos de quienes ya están ocupados. El siguiente listado ilustra el nivel profesionalizante de la carrera: tributación, con $84 \%$; auditoría, con $83.1 \%$; contabilidad general, con $82.2 \%$;

contabilidad financiera, con 72.9\%; contabilidad de costos, con $71.2 \%$; manejo de modelos contables, con $68.6 \%$; contabilidad para la gerencia, con $66.9 \%$, y contabilidad gubernamental, con $44.1 \%$. El conocimiento contable requiere de una compresión global y sistémica de los problemas, con interacciones de conocimientos, destrezas, procesos y donde se valore el enfoque disciplinar, pero con posibilidades a otros niveles superiores de carácter transdisciplinar para enriquecer la profesión (Casal, 2011).

Entre las materias auxiliares de la carrera, solo figuran muy pocas, por ejemplo, Metodología de la Investigación con el $39.8 \%$, le sigue Emprendimiento con el $37.3 \%$; Marketing con el $28 \%$ y escasamente la Lógica Matemática con el $20.3 \%$ de importancia. De las 52 disciplinas recibidas en la carrera, estas configuran el perfil de egreso en cuanto a la formación y son consideradas como significativas y necesarias, esto deja 
una ventana abierta para que las autoridades de la facultad y los órganos de control académico reconfiguren la profesión en términos de mejoramiento de contenidos, metodologías, procesos, enfoques de enseñanza y paradigmas educativos en pro de la carrera del contadorauditor, como bien lo dice Dextre (2013):

El egresado de la carrera de contabilidad que cuente con competencias desarrolladas durante su formación debe estar preparado para desempeñarse en ocupaciones de la profesión en espacios como empresas nacionales e internacionales, sociedades de auditoría, organismos reguladores y de control, entidades públicas, instituciones financieras, estudios contables, entre otros. (p. 40)

La pregunta nuclear de esta investigación es el nivel de formación lograda en investigación científica y las respuestas son como sigue: poco, $14.4 \%$; normal, $63.6 \%$; bastante, $15.3 \%$; mucho, $6.8 \%$. Lo esencial es cuánto de esos conocimientos se han puesto en práctica durante la carrera y si su aprendizaje servirá a futuro en su vida laboral. No basta solo con dotar del conocimiento sino su aplicación como factor de transformación de una realidad y como recurso para alcanzar mejores desempeños.

Los valores anteriores contrastan con la satisfacción de formación recibida durante la carrera y sus respuestas dejan estas cifras: completamente insatisfecho, $4.2 \%$; insatisfecho, $10.2 \%$; neutral, $22.9 \%$; satisfecho, $53.4 \%$, y completamente satisfecho, $9.3 \%$. Al aplicar una prueba de Ji cuadrado $\left(\mathrm{X}^{2}(21,02)=16,289 ; p=0,178 ; \mathrm{gl}=12\right)$, se evidencia que no hay diferencia estadística significativa. Esto significa que igual da recibir o no recibir investigación científica en la carrera; solo están más preocupados de las asignaturas que le agregan valor a su formación y son las de especialidad o profesionalizantes. 
Finalmente, se verificó algunos indicadores que evidencian su desarrollo profesional y se buscó su correlación interna como se presenta en la tabla 1.

Tabla 1 Correlación de indicadores de desarrollo profesional

\begin{tabular}{|c|c|c|c|c|c|c|c|c|c|}
\hline & & P1 & P2 & P3 & P4 & P5 & P6 & P7 & P8 \\
\hline \multirow{2}{*}{ P2 } & $r$ & $.763^{* *}$ & & & & & & & \\
\hline & Sig. Bil. & .000 & & & & & & & \\
\hline \multirow{2}{*}{ P3 } & $r$ & $.780^{* *}$ & $.858^{* *}$ & & & & & & \\
\hline & Sig. Bil. & .000 & .000 & & & & & & \\
\hline \multirow{2}{*}{ P4 } & $r$ & $.746^{* *}$ & $.818^{* *}$ & $.773^{* *}$ & & & & & \\
\hline & Sig. Bil. & .000 & .000 & .000 & & & & & \\
\hline \multirow{2}{*}{ P5 } & $r$ & $.593^{* *}$ & $.793^{* *}$ & $.786^{* *}$ & $.760^{* *}$ & & & & \\
\hline & Sig. Bil. & .000 & .000 & .000 & .000 & & & & \\
\hline \multirow{2}{*}{ P6 } & $r$ & $.655^{* *}$ & $.776^{* *}$ & $.800^{* *}$ & $.745^{* *}$ & $.823^{* *}$ & & & \\
\hline & Sig. Bil. & .000 & .000 & .000 & .000 & .000 & & & \\
\hline \multirow{2}{*}{ P7 } & $r$ & $.670^{* *}$ & $.747^{* *}$ & $.779^{* *}$ & $.696^{* *}$ & $.767^{* *}$ & $.815^{* *}$ & & \\
\hline & Sig. Bil. & .000 & .000 & .000 & .000 & .000 & .000 & & \\
\hline \multirow{2}{*}{ P8 } & $r$ & $.730^{* *}$ & $.863^{* *}$ & $.787^{* *}$ & $.875^{* *}$ & $.814^{* *}$ & $.805^{* *}$ & $.770^{* *}$ & \\
\hline & Sig. Bil. & .000 & .000 & .000 & .000 & .000 & .000 & .000 & \\
\hline \multirow{2}{*}{ P9 } & $r$ & $.754^{* *}$ & $.825^{* *}$ & $.819^{* *}$ & $.810^{* *}$ & $.759^{* *}$ & $.741^{* *}$ & $.801^{* *}$ & $.893^{* *}$ \\
\hline & Sig. Bil. & .000 & .000 & .000 & .000 & .000 & .000 & .000 & .000 \\
\hline
\end{tabular}

Tabla 2 Codificación de indicadores

\begin{tabular}{|c|c|}
\hline \multicolumn{2}{|r|}{ Desarrollo y formación de los estudiantes } \\
\hline P1 & Tiene la oportunidad de desarrollarse profesionalmente fuera de la universidad \\
\hline P2 & Puede aplicar los conocimientos adquiridos en el ámbito laboral \\
\hline P3 & $\begin{array}{c}\text { Ha desarrollado habilidades y competencias que le permitan desarrollarse en el campo } \\
\text { profesional }\end{array}$ \\
\hline P4 & Ha desarrollado una ética de actuación profesional \\
\hline P5 & El contenido de las asignaturas ha sido útil para su formación profesional \\
\hline
\end{tabular}

Revista Activos

ISSN: 0124-5805 | e-ISSN: 2500-5278 | DOI: https://doi.org/10.15332/25005278

Vol. 19 N.o 1 | enero-junio de 2021 


\begin{tabular}{|c|c|} 
Desarrollo y formación de los estudiantes \\
\hline P6 & La formación recibida está cumpliendo con los objetivos de la carrera \\
\hline P7 & Capacidad de interpretar leyes y reglamentos \\
\hline P8 & Capacidad de trabajar en equipo \\
\hline P9 & Capacidad para proponer, ayudar y colaborar con alternativas para la adecuada toma de \\
decisiones
\end{tabular}

Fuente: elaboración propia.

Desde el punto de vista de asociación de las variables en análisis sobre el desarrollo profesional, el indicador P1, sobre la oportunidad de desarrollarse profesionalmente fuera de la universidad, presenta correlación media con los indicadores sobre la utilidad de las asignaturas y su impacto en la formación profesional ( $\left.\mathrm{P}_{5}\right)(r=0,593)$; del mismo modo con el indicador sobre los objetivos de la carrera (P6) $(r=0,655)$ y finalmente con la capacidad de interpretación de leyes y reglamentos $\left(\mathrm{P}_{7}\right)$ $(r=0,670)$. La evidencia demuestra que, siendo una correlación media, presenta debilidades que se traslada desde el aula de clase hacia el campo laboral como una falencia en la formación recibida en los espacios universitarios. Queda la responsabilidad en la institución educativa de fortalecer el pénsum, los contenidos, los objetivos curriculares, las metodologías de enseñanza aprendizaje, orientadas a formar un profesional contable para el siglo XXI.

Así mismo, otro grupo de indicadores presenta correlaciones fuertes; por ejemplo, la capacidad de trabajo en equipo (P8) está muy bien asociada con los conocimientos adquiridos en el campo laboral (P2) y con la ética de actuación profesional; el valor más significativo está asociado con la capacidad que tiene el profesional contable para proponer, ayudar y colaborar con alternativas para la adecuada toma de decisiones $(r=0,893)$. 
Tabla 3. Correlación de las capacidades adquiridas en la formación profesional

\begin{tabular}{|c|c|c|c|c|c|c|c|c|c|c|c|c|}
\hline & & P1 & P2 & P3 & P4 & P5 & P6 & P7 & P8 & P9 & P10 & P11 \\
\hline \multirow[b]{2}{*}{ P2 } & $r$ & $.587^{* *}$ & & & & & & & & & & \\
\hline & $\begin{array}{l}\text { Sig. } \\
\text { Bil. }\end{array}$ & .000 & & & & & & & & & & \\
\hline \multirow[b]{2}{*}{ P3 } & $r$ & $.696^{* *}$ & $.595^{* *}$ & & & & & & & & & \\
\hline & $\begin{array}{l}\text { Sig. } \\
\text { Bil. }\end{array}$ & .000 & .000 & & & & & & & & & \\
\hline \multirow{2}{*}{ P4 } & $r$ & $.691^{* *}$ & $.621^{* *}$ & $.741^{* *}$ & & & & & & & & \\
\hline & $\begin{array}{l}\text { Sig. } \\
\text { Bil. }\end{array}$ & .000 & .000 & .000 & & & & & & & & \\
\hline \multirow[b]{2}{*}{ P5 } & $r$ & $.694^{* *}$ & $.667^{* *}$ & $.720^{* *}$ & $.694^{* *}$ & & & & & & & \\
\hline & $\begin{array}{l}\text { Sig. } \\
\text { Bil. }\end{array}$ & .000 & .000 & .000 & .000 & & & & & & & \\
\hline \multirow{2}{*}{ P6 } & $r$ & $.679^{* *}$ & $.584^{* *}$ & $.758^{* *}$ & $.632^{* *}$ & $.771^{* *}$ & & & & & & \\
\hline & $\begin{array}{l}\text { Sig. } \\
\text { Bil. }\end{array}$ & .000 & .000 & .000 & .000 & .000 & & & & & & \\
\hline \multirow[b]{2}{*}{ P7 } & $r$ & $.641^{* *}$ & $.623^{* *}$ & $.739^{* *}$ & $.655^{* *}$ & $.746^{* *}$ & $.692^{* *}$ & & & & & \\
\hline & $\begin{array}{l}\text { Sig. } \\
\text { Bil. }\end{array}$ & .000 & .000 & .000 & .000 & .000 & .000 & & & & & \\
\hline \multirow[b]{2}{*}{ P8 } & $r$ & $.713^{* *}$ & $.619^{* *}$ & $.755^{* *}$ & $.690^{* *}$ & $.713^{* *}$ & $.709^{* *}$ & $.758^{* *}$ & & & & \\
\hline & $\begin{array}{l}\text { Sig. } \\
\text { Bil. }\end{array}$ & .000 & .000 & .000 & .000 & .000 & .000 & .000 & & & & \\
\hline \multirow[b]{2}{*}{ P9 } & $r$ & $.626^{* *}$ & $.624^{* *}$ & $.723^{* *}$ & $.640^{* *}$ & $.704^{* *}$ & $.690^{* *}$ & $.750^{* *}$ & $.814^{* *}$ & & & \\
\hline & $\begin{array}{l}\text { Sig. } \\
\text { Bil. }\end{array}$ & .000 & .000 & .000 & .000 & .000 & .000 & .000 & .000 & & & \\
\hline \multirow{2}{*}{ P10 } & $r$ & $.637^{* *}$ & $.644^{* *}$ & $.673^{* *}$ & $.694^{* *}$ & $.649^{* *}$ & $.654^{* *}$ & $.737^{* *}$ & $.740^{* *}$ & $.780^{* *}$ & & \\
\hline & $\begin{array}{l}\text { Sig. } \\
\text { Bil. }\end{array}$ & .000 & .000 & .000 & .000 & .000 & .000 & .000 & .000 & .000 & & \\
\hline \multirow[b]{2}{*}{ P11 } & $r$ & $.559^{* *}$ & $.541^{* *}$ & $.686^{* *}$ & $.655^{* *}$ & $.683^{* *}$ & $.590^{* *}$ & $.670^{* *}$ & $.617^{* *}$ & $.717^{* *}$ & $.635^{* *}$ & \\
\hline & $\begin{array}{l}\text { Sig. } \\
\text { Bil. }\end{array}$ & .000 & .000 & .000 & .000 & .000 & .000 & .000 & .000 & .000 & .000 & \\
\hline \multirow[b]{2}{*}{ P12 } & $r$ & $.654^{* *}$ & $.682^{* *}$ & $.644^{* *}$ & $.657^{* *}$ & $.661^{* *}$ & $.555^{* *}$ & $.694^{* *}$ & $.722^{* *}$ & $.715^{* *}$ & $.746^{* *}$ & $.684^{* *}$ \\
\hline & $\begin{array}{l}\text { Sig. } \\
\text { Bil. }\end{array}$ & .000 & .000 & .000 & .000 & .000 & .000 & .000 & .000 & .000 & .000 & .000 \\
\hline
\end{tabular}

Fuente: elaboración propia.

Revista Activos

ISSN: 0124-5805 | e-ISSN: 2500-5278 | DOI: https://doi.org/10.15332/25005278

Vol. 19 N. ${ }^{\circ} 1$ | enero-junio de 2021 
Tabla 4. Codificación de las capacidades

\begin{tabular}{|r|r|}
\hline P1 & \multicolumn{1}{c}{ Codificación } \\
\hline P2 & Manejo de normas y principios de contabilidad, así como de teoría contable \\
\hline P3 & Desarrollo de pensamiento crítico, creativo y complejo \\
\hline P4 & Capacidad para elaborar, analizar e interpretar estados financieros \\
\hline P5 & Posee capacidad de reconocer y resolver problemas complejos como estrategia didáctica \\
\hline P6 & Manejar la metodología estadística-matemática en el área contable, finanzas y costos \\
\hline P7 & Capacidad para comprender y analizar la estructura financiera de una entidad \\
\hline P8 & Actualización en temas de normativa legal y laboral \\
\hline P9 & Capacidad de análisis e interpretación de la normativa tributaria \\
\hline P10 & Responsabilidad social \\
\hline P11 & Manejo de softwares contables para el registro de datos y hechos económicos \\
\hline P12 & Capacidad para tomar iniciativas propias para su desarrollo profesional \\
\hline
\end{tabular}

Fuente: elaboración propia.

El análisis correlacional en cuanto a las capacidades adquiridas en la formación profesional en la academia, muestra que las de mayor puntuación están asociadas entre la capacidad para comprender y analizar la estructura financiera de una entidad con la actualización en temas de normativa legal y laboral $(r=0,758)$. Otro par de indicadores moderadamente asociados son la capacidad de análisis e interpretación de la normativa tributaria con la actualización que se pueda tener en el mismo campo $(r=0,814)$, que se asocia con la responsabilidad social del profesional $(r=0,780)$.

Una correlación media $(r=0,541)$ se da entre los indicadores de desarrollo del pensamiento crítico y complejo con el manejo de software contables para el registro de datos y hechos económicos. Es posible que se deba a que las transacciones se lo realizan de manera mecánica en función de la estructura del paquete contable que no da oportunidad a la reflexión, la crítica y el análisis. 


\section{Conclusiones}

La investigación no es una fortaleza en la formación de los estudiantes de la carrera de contabilidad y auditoría, debido a que la malla curricular es mayormente profesionalizante, razón que justifica la preferencia por asignaturas que están directamente relacionadas con su perfil profesional. Los estudiantes de la carrera de Contabilidad y Auditoría aplican la investigación, en su mayoría, en la elaboración de proyectos. Esto se explica en vista de que una de las modalidades de graduación con mayor aceptación son los proyectos de investigación, lo que demuestra que no existe cultura en investigación a nivel de pregrado.

La investigación contable es un tema poco claro y confuso para los estudiantes de la carrera; lo consideran como el estudio del comportamiento de eventos financieros y de la teoría contable en su mayoría. De esta manera, se evidencia una vez más que los estudiantes de la carrera no poseen una sólida formación en temas de investigación.

Las asignaturas que mayor influencia tuvieron en la formación profesional de los estudiantes de la carrera fueron Tributación, Auditoría y Contabilidad, materias que están directamente relacionadas con su desempeño laboral. De las asignaturas auxiliares, es importante resaltar que Metodología de la Investigación es considerada como la de mayor influencia, aunque su uso no sea significativo.

\section{Referencias}

Bahía, B. (2004). Reflexiones sobre abordajes científicos en la investigación contable. Escritos Contables, (45). http://bibliotecadigital.uns.edu.ar/scielo.php?script=sci arttext\&pid=S151442752004001100003\&lng=en\&nrm=iso\&tlng=es\&ORIGINALLANG=es 
Cabeza, L. y Castillón, J. (2005). Necesidades de las bases conceptuales contables del área financiera en los estudiantes de Administración de Empresas. Pensamiento \& Gestión, 18, 69-102. https://www.redalyc.org/pdf/646/64601804.pdf

Casal, R. A. (julio-diciembre de 2011). De la formación disciplinar a la transdisciplinar del contador público. Visión Gerencial, (2), 273-282. https://www.redalyc.org/pdf/4655/465545891004.pdf

Casal, R. y Viloria, N. (2007). La ciencia contable, su historia, filosofía, evolución y su producto. Actualidad Contable Faces, 1O(15), 19-28. https://www.redalyc.org/pdf/257/25701503.pdf

Castro, R. (2016). Teoría contable (3.a ed.). Remington FER.

Cervantes, C., Samaniego, P. y Aguilar, V. (2018). Reflexión sobre la historia y evolución de la ciencia contable. Arjé. Revista de Postgrado FACE-UC, 12(22), 275-282. http://www.arje.bc.uc.edu.ve/arje22e/art26.pdf

Cifuentes, J. E. y Pedraza, J. I. (2017). Importancia de la investigación en la formación de estudiantes en la modalidad a distancia. Revista Educación y Humanismo, 19(32), 31-52. http://dx.doi.org/10.17081/eduhum.19.32.2530

Colina, L. (2007). La investigación en la educación superior y su aplicabilidad social. Revista de educación LAURUS, 13(25), 330-353. https://www.redalyc.org/pdf/761/76111479016.pdf

Fernández, C., Pelekais, E. A. y Farfán, C. (2019). Perfil del contador público desde su ámbito de formación profesional. Global Negotium, 3(1), 42-57. http://publishing.fgu-edu.com/ojs/index.php/RGN/article/view/120/150

Gallegos, C. y Rodríguez, E. (2017). Formación contable en Chile.Una propuesta de articulación curricular. Actualidades Investigativas en Educación, 17(3), 1-32. http://dx.doi.org/10.15517/aie.v17i3.29988

Gómez, M. (2003). Algunos comentarios sobre la potencialidad de la investigación en contabildad. INNOVAR, 21, 139-144. http://www.scielo.org.co/pdf/inno/v13n21/v13n21a11.pdf

Gómez, Y. (2013). Enseñabilidad y educabilidad.Nuevas tendencias en la enseñanza y la investigación contables. En-Contexto, 1(1), 179-192. https://www.redalyc.org/pdf/5518/551856274010.pdf 
González, G. (2003). Contabilidad General. Ediciones Académicas.

González, P. y Bermúdez, T. (2010). Fuentes de información, indicadores y herramientas más usadas por gerentes de Mipyme en Cali, Colombia. Contaduría y Administración(232), 83-108. http://www.scielo.org.mx/pdf/cya/n232/n232a5.pdf

Hernandez, R., Fernández, C. y Baptista, M. P. (2014). Metodología de la investigación (6. ${ }^{\mathrm{a}}$ ed.). Mc Graw Hill.

Macías, H. A. (2,3 y 4 de Octubre de 2013). Investigación contable interpretativa, enfoque alterno a la corriente principal con alto potencial para Latinoamérica. Recuperado el 16 de junio de 2020, de http://congreso.investiga.fca.unam.mx/docs/xviii/docs/16.06.pdf

Mantilla-Falcón, L. M., Galarza-Galarza, J. C. y Zamora-Sánchez, R. A. (2017). La inserción de la mujer en la educación superior ecuatoriana: caso Universidad Técnica de Ambato. Revista Latinoamericana de Estudios Educativos, 13(2), 1229. https://doi.org/10.17151/rlee.2017.13.2.2

Mantilla-Falcón, M., Tobar-Vasco, G. H., Arias-Pérez, M. G., \& Ríos-Urrutia, G. C. (2018). Competencias del contador-auditor en el perfil de egreso. Caso Universidad Técnica de Ambato, Ecuador. Actualidad Contable FACES, 21(37), 90-117. Recuperado el 04 de junio de 2020, de https://www.redalyc.org/articulo.oa?id=25755483005

Merino, J. L., Parrales, J., Figuero, M. y Alvarez, A. (2019). Principios, convenciones, doctrinas y normas de la contabilidad. Universidad Estatal del Sur de Manabí. http://doi.org/10.17993/EcoOrgyCso.2019.56

Ordoñez-Noreña, S. L. (2008). Contra el adiestramiento contable: invitación a la ruptura epistemológica en la formación del contador público. En D. Ariza (dir.) Perspectivas críticas de la contabilidad. Reflexiones y críticas (pp. 117-127). Universidad Nacional de Colombia.

Ortiz-Ocaña, A. L. (2016). Introducción a la investigación contable. Ediciones Universidad Cooperativa de Colombia. http://dx.doi.org/10.16925/greylit.1370

Pérez, V. y Pinto Perry , G. (2016). La investigación contable como herramienta didáctica en la docencia de la contabilidad. Revista Digital FCE, 7, 37-47. https://revistas.unlp.edu.ar/CADM/article/download/2037/2457/ 
Pinzón, J. E. (2011). Historia de la contaduría pública en Colombia siglo XX a 20 años de su publicación. Cuadernos de Contabilidad, 12(30), 368-380.

http://www.scielo.org.co/pdf/cuco/v12n3o/v12n30a15.pdf

Quinche, F. L. (2006). Historia de la contabilidad, una revisión de las perspectivas tradicionales y críticas de historiografía contable. Revista Facultad de Ciencias Económicas Investigación y Reflexión, 14(1), 187-201. https://www.redalyc.org/pdf/909/90900110.pdf

Quintanilla, A. L. (2008). La producción de conocimiento en América Latina. Salud Colectiva, 4(3), 253-260. https://10.18294/sc.2008.344

Ríos, R. A. (2012). La formación para la investigación de contadores públicos colombianos: un asunto de estrategia. Revista Científica General José María Córdova, 11(11), 175-206. http://www.scielo.org.co/pdf/recig/v11n11/v11n11aog.pdf

Saavedra, M. L. y Saavedra, M. E. (2015). La investigación contable en Latinoamérica. Actualidad Contable Faces, 18(31), 99-121. https://www.redalyc.org/pdf/257/25743363006.pdf

Segarra, H. P., Hidalgo, W. A. y Montalvan, J. A. (2016). Metodología de las investigaciones en Contabilidad. Revista Publicando, 3(9), 106-715. https://dialnet.unirioja.es/descarga/articulo/5879390.pdf

Villareal, J. L. y Córdoba, J. X. (2017). Fundamentos para el Estudio de las corrientes de investigación en contabilidad. Tendencias, 18(2), 139-151.

http://dx.doi.org/10.22267/rtend.171802.81 